\title{
Priority access for QoS support in distributed wireless networks
}

\author{
ZHOU Xin* and ZHENG Changwen
}

Institute of Software, Chinese Academy of Sciences, Beijing 100190, China

\begin{abstract}
Guaranteed quality of service (QoS) support has been an open issue of distributed wireless networks for years. The IEEE 802.11 e provides a valuable method for this purpose. However, it could only provide service differentiation, rather than service guarantee, for multi-priority traffic. Many studies have tried to improve its QoS ability, but still leave some problems. This paper investigates these problems and proposes a scheme called the priority access based on busy tone (PABT) to improve the QoS performance. To guarantee the priority channel access, this scheme uses an in-band busy tone to limit the transmission of lower-priority traffic when higher-priority traffic has packets to send. Based on that, it also optimizes the contention window tuning according to the flows number of each traffic type individually, in order to improve the throughput as well as the traffic capacity. Simulation results show that the proposed scheme significantly improves the real-time traffic capacity, throughput, delay, fairness and packet loss rate.
\end{abstract}

Keywords: $802.11 \mathrm{e}$, quality of service (QoS), busy tone, priority access, traffic capacity, contention window.

DOI: $10.21629 /$ JSEE.2019.06.14

\section{Introduction}

With the development of mobile Internet technology, the volume of the multimedia traffic, i.e., voice, video and online games, grows rapidly by about $20 \%$ per year [1]. Unlike the traditional data service, the multimedia traffic requires much stricter quality of service (QoS) requirements such as a maximal delay, a minimal throughput, and a bounded packet loss rate (PLR).

It is not so easy to support QoS in wireless networks, because the signal transmission may suffer from the interference, multi-path fading and shadow effect, which will result in high PLR and delay jitter. If the communicating devices are within an effective range and the channel conditions are good enough, the QoS performance will typically rely on the channel access method [2].

Manuscript received August 13, 2018.

*Corresponding author.

This work was supported by the Special Fund for National Defense Technology Innovation (18-163-11-ZT-003-027-01).
The 802.11e enhanced distributed channel access (EDCA) [3] is one of the most widely used channel access methods for QoS support in distributed wireless networks. According to the traffic types, frames from upper layers are divided into four access categories (ACs), which are AC_VO, AC_VI, AC_BE and AC_BK for voice, video, best-effort, and background traffic respectively. To differentiate the QoS, the EDCA uses a set of AC specific parameters such as contention window $(\mathrm{CW})$ and arbitration inter-frame space (AIFS). It assigns smaller $\mathrm{CW}$ ranges and AIFS numbers (AIFSN) to higher-priority (HP) ACs for more access probabilities, as shown in Table 1.

Table 1 Some QoS parameters for the 802.11e EDCA

\begin{tabular}{cccc}
\hline AC & CWmin & CWmax & AIFSN \\
\hline AC_VO & $(\mathrm{aCWmin}+1) / 4-1$ & $(\mathrm{aCWmin}+1) / 2-1$ & 2 \\
AC_VI & $(\mathrm{aCW}$ min+1)/2-1 & $\mathrm{aCWmin}$ & 3 \\
AC_BE & $\mathrm{aCWmin}$ & $\mathrm{aCWmax}$ & 6 \\
AC_BK & $\mathrm{aCWmin}$ & $\mathrm{aCWmax}$ & 9 \\
\hline
\end{tabular}

Even if the EDCA algorithm provides effective QoS differentiation support for 802.11 networks, it suffers from several problems widely investigated in previous studies [4-8]. First, with the growth of the network size, the throughput performance will decline as the collision rate increases. This is the legacy network size problem encountered in traditional 802.11. Second, the HP traffic cannot be supported well when there is much lower-priority (LP) traffic in the network. Although the AIFSN of the HP traffic is smaller than that of the LP, the LP traffic can still transmit packets prior to the HP if the former's random backoff interval after the AIFS phase is much shorter than the latter [8]. This is the called uncertain priority access problem. Third, the EDCA can only support a limited number of concurrent real-time traffic flows, due to their small $\mathrm{CW}$ sizes, which give them higher priority but may result in more collisions. We call it the limited real-time traffic capacity problem.

Many algorithms enhancing the QoS ability of the 802.11e has been studied [9-17]. Reference [9] aims to provide the weighted fair share of the throughput among 
different applications, rather than the privileged access for the HP traffic. The 802.11aa standard [10] extends the granularity of the traffic type to provide differentiation between individual video streams, but it does not change the access rules. The authors of [11] and [12] utilized the queue-adaptive scheduling schemes to employ differentiation among the same AC. The scheme in [13] gave several real-time flows a privilege of immediate access to the channel after the AIFS phase, so as to guarantee QoS support; however, it completely neglects others' service. The prioritized idle sense (PIS) algorithm [14] adjusts the CW size according to a smaller target $n_{i}^{\alpha}$ for the HP traffic $i$ and a larger value for the LP traffic, but it can only differentiate a few ACs in the case of no significant reduction in throughput. Above all, the QoS support is usually provided by changing the AC specific parameters like AIFS and $\mathrm{CW}$.

This paper proposes a scheme called the priority access based on busy tone (PABT) for distributed wireless networks. It uses an in-band slot-length busy tone (BT) signal, i.e., sine signal, to reserve the channel for absolute priority access. Based on that, it also improves the realtime traffic capacity and the aggregated throughput significantly through the optimal CW tuning for each traffic type individually.

The rest of this paper is organized as follows. Section 2 introduces the related work mainly about the BT and the $\mathrm{CW}$ tuning schemes. Section 3 describes the proposed PABT scheme and the analytical model in detail. Section 4 presents the simulation results, and the conclusion is drawn in Section 5.

\section{Related work}

There is a considerable amount of research on QoS support for distributed wireless networks. We discuss here only a small subset of closely related work, especially on the BT scheme and the optimal $\mathrm{CW}$ tuning algorithm.

The BT scheme was first proposed to solve the hidden and exposed terminal problems in multi-hop networks $[18,19]$. To the best of our knowledge, literature [20] is the first research that introduces the BT scheme into QoS support. It uses BT signals to reserve the priority access for real-time traffic, but can only differentiates two traffic priorities. Contention tone protocol (CTP) [21] uses one separate control channel to transmit BT for channel contention. By diverting the contention overhead to the control channel, CTP achieves high data channel utilization, but it costs an additional control channel and transceiver, and remains suffering the EDCA's problems. Jiang's method [22] improves the spectrum efficiency by using the in-band BT signal instead of the out-of-band, but the access delay is larger since the station with the largest backoff interval will have priority access to the channel. The deterministic priority channel access (DPCA) scheme [23] addresses this deficiency by the transmission of BT at the end of the AIFS phase, but it still follows the traditional methods to set and tune the $\mathrm{CW}$ sizes, which leaves the limited realtime traffic capacity problem unresolved. The in-band BT scheme [24] uses a similar method with the real-time traffic capacity problem mentioned, but the $\mathrm{CW}$ adjustment is not optimized.

The normal CW tuning methods, i.e., EDCA, generally increase the $\mathrm{CW}$ size when the sender catches a collision, and decrease the $\mathrm{CW}$ size when transmission succeeds. Although they are simple and widely used, the performance is not so satisfying, because they only adjust the $\mathrm{CW}$ size based on the collision status of the sender itself. The optimal CW tuning algorithms generally tune the $\mathrm{CW}$ size dynamically according to the number of active nodes $[25,26]$ or the number of consecutive idle backoff slots [27-30] for maximal throughput and fairness, but these algorithms are not suitable for the QoS support among the multipriority traffic flows.

Gao et al. gave a detailed analysis on the modeling and optimization of the 802.11e EDCA network in [31]. They prove that the QoS differentiation can be met by adjusting the increasing rate of the $\mathrm{CW}$ size of each $\mathrm{AC}$ according to the expected ratio of throughput, and then propose a CW tuning method based on the modified beacon frame transmitted by the access point. However, this method focuses on providing the proportional throughput allocation, not the absolute priority access. The priority-based fair medium access control (P-MAC) protocol [32] adjusts the $\mathrm{CW}$ size according to relative weights among the traffic flows and contention levels of a wireless medium, but this method is too sensitive to converge to the optimal operating point. Nassiri et al. proposed the PIS scheme [14] for QoS support based on the Idle-sense algorithm [27]. In the PIS, each station adjusts its CW size based on the observed average number of idle slots. It sets the target number of the idle slots to a small value $n_{i}^{\alpha}$ for the HP traffic $i$, in order to make the HP traffic get a smaller $\mathrm{CW}$ size and then gain more access probability. However, the empirical setting for this parameter will break down the optimal performance of the original idle-sense algorithm. In addition, the PIS can only differentiate a few ACs in the case of no significant reduction in throughput.

Although much research has tried to address the problems of QoS support in distributed wireless networks, no method proposed so far offers all the desirable characteristics simultaneously: large capacity for real-time traffic, guaranteed support for priority access, high throughput for various contention levels, and fair access for the flows with the same priority. 


\section{PABT scheme}

In this section, we describe the proposed PABT scheme in detail, including the usage of the BT and the CW tuning algorithm.

\subsection{BT reservation}

The BT reservation for priority access is the most important feature of the PABT scheme. Fig. 1 compares the access process of the 802.11e EDCA and the proposed PABT scheme. They are very similar, except for the BT transmission at the end of the AIFS phase. In PABT, the sender will transmit an in-band BT signal at the last slot of the AIFS phase, which will keep silent in the 802.11e EDCA. Any stations that detect the BT signal during their AIFS phases will defer until the subsequent packet transmission completes. Hence, the BT signal works like a deterministic priority reservation. As long as one HP flow exists, all LP flows will sense the BT signal of the HP flow before sending their own and defer then. This scheme ensures that the HP traffic flow always access the channel prior to the LP without any doubt. If two or more traffic flows have the same priority, they will transmit BT signals concurrently without sensing each other, but the succeeding backoff phase will still help to avoid collisions. Short inter-frame space (SIFS) is a predefined time parameter in the IEEE 802.11 Standard determined by the Physical (PHY) layer, and ACK is a predefined medium access control (MAC) frame in the IEEE 802.11 Standard to reply for the received Data frame.

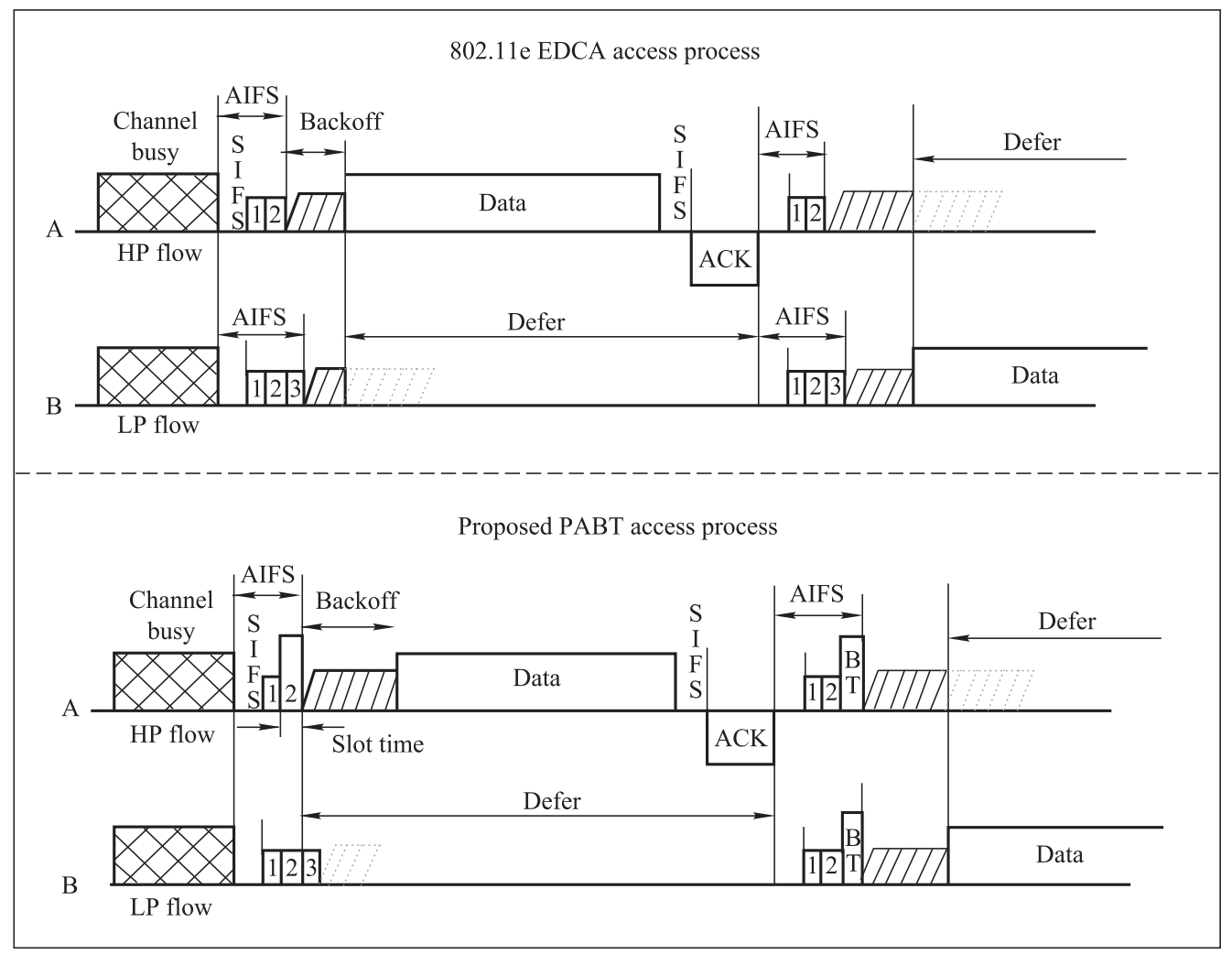

Fig. 1 Comparison of the access process between EDCA and PABT

For proper operation, the AIFSN of the HP traffic should be given a smaller value, i.e.,

$$
\left\{\begin{array}{l}
\operatorname{AIFSN}[i]=\mathrm{AIFSN}[i-1]+1, \quad 1 \leqslant i \leqslant A C_{\max } \\
\mathrm{AIFSN}[0]=2 \\
\mathrm{AIFS}=\mathrm{SIFS}+\mathrm{AIFSN} \cdot \operatorname{slot}
\end{array}\right.
$$

where a smaller $i$ indicates higher priority, and $A C_{\max }$ indicates the largest value of $i$.

For the simplicity of transmission and detection, the BT should be a narrow band signal. As shown in Fig. 2, the same transmitting power will be concentrated in a much narrower band during the busy tone transmission, and thus a spectrum spike at the BT frequency point will still be very clear even if there is some interference from unexpected 802.11 stations.

We recommend the sine signal as the BT, due to its simplicity and good performance on detection sensitivity and time cost. In addition, collisions between sine signals cannot change their spectrum characteristics, and thus do not affect the detection results. Based on the discussion in [21], a detection time of $5 \mu$ s is sufficient in such a case. 
A simple test has been implemented to verify this conclusion through Matlab simulator. In this simulation, the fast Fourier transformation (FFT) is used for single tone detection with the receiver bandwidth $20 \mathrm{MHz}$, the sample frequency $50 \mathrm{M}$ sample/s, and the detection time $2 \mu \mathrm{s}$. As shown in Fig. 3, the spectrum spike of the single tone is very clear even when $\mathrm{SNR}=-5 \mathrm{~dB}$, and thus can be easily detected. To be mentioned, in Fig. 3, the BT is transmitted by one station and the noise may be generated by other station's data transmission.

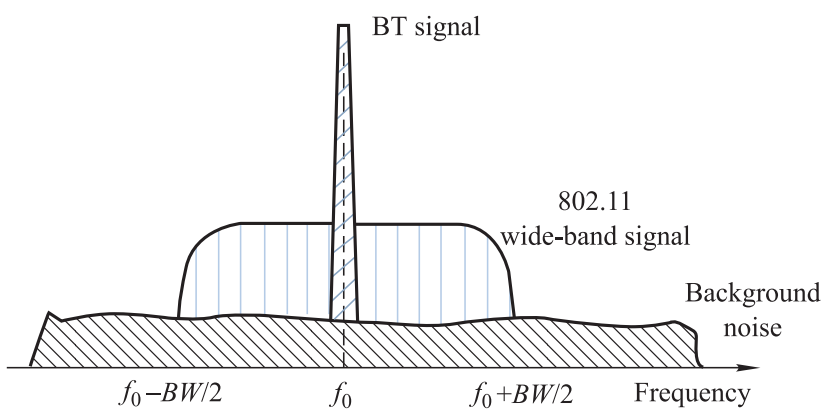

Fig. 2 Spectrum sketch map of in-band BT

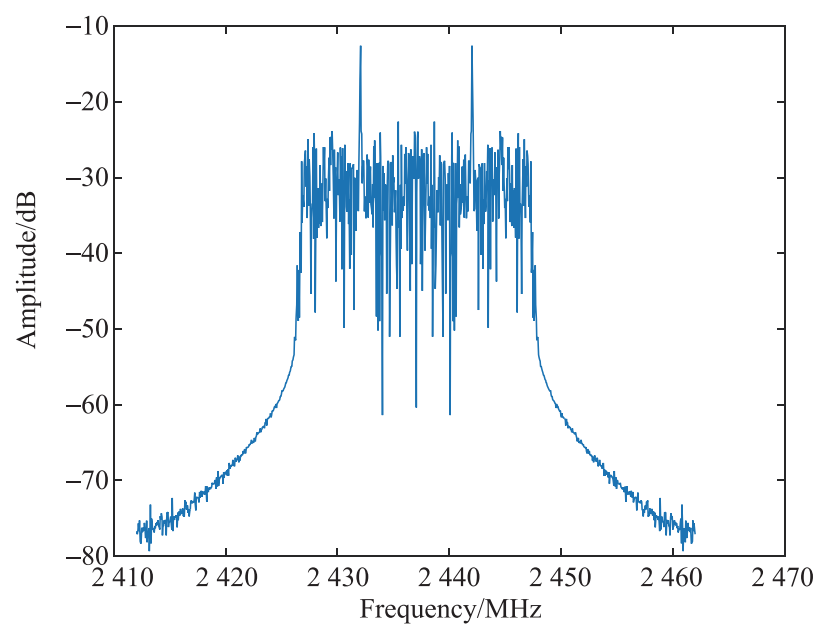

Fig. 3 FFT for BT detection (detection time $=2 \mu \mathrm{s}, \mathrm{SNR}=-5 \mathrm{~dB}$ )

To further investigate the detection performance for the BT, we implement more simulations with an intuitive rule: if the maximal amplitude of FFT spikes locates on the predefined frequency of the BT, and its value is $6 \mathrm{~dB}$ higher than the average amplitude, we assert that a BT is detected. This detection rule can be expressed as follows:

$$
\left\{\begin{array}{l}
f_{b}=\arg \max \{A(f)\} \\
A\left(f_{b}\right) \geqslant A_{\text {mean }}+6
\end{array}\right.
$$

where, $f_{b}$ is the predefined frequency of the BT, $A_{\text {mean }}$ is the average amplitudes of the in-band FFT spikes, and $A\left(f_{b}\right)$ is the amplitude of the FFT spike on $f_{b}$. Fig. 4 shows that the BT detection probability is nearly $100 \%$ even if the SNR is as low as $-12 \mathrm{~dB}$. In Fig. 4, the performance of three threshold values are compared. The similar results show that the first line in (2) is the deterministic factor and the value of threshold produces little effect.

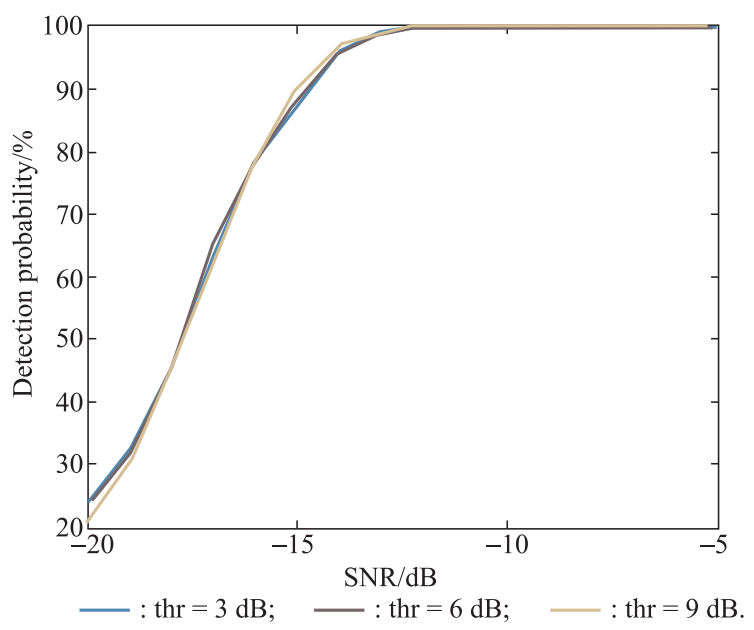

Fig. 4 FFT detection performance for BT

Although the access process of the PABT scheme is only described in the basic Data/ACK mode in this section, it is worth mentioning that it can also be applied to the ready to send/clear to send (RTS/CTS) mode, since it only modifies the common process before any frame transmission. Furthermore, the RTS/CTS mode is more recommended in a multi-hop environment to alleviate the hidden terminal problem.

\subsection{Individually optimized CW tuning}

As shown in Table $1, C W_{\max }$ of the HP traffic is generally less than $C W_{\min }$ of the LP traffic in EDCA, in order to distinguish the priorities. However, this is not necessary for PABT, since the deterministic priority access has already been provided by the BT scheme. As a result, the $\mathrm{CW}$ sizes of each $\mathrm{AC}$ can be individually optimized according to their own conditions. This is the core idea to solve the limited real-time traffic capacity problem.

In this section, we first introduce our analytical model for the optimal CW setting in the single AC scenario, which was proposed earlier in [30]. Then, we propose a dynamic $\mathrm{CW}$ tuning algorithm, which can be extended to cover the multi-priority AC scenarios under the premise of guaranteed priority access.

Suppose $M$ stations in the network compete for the channel at the same time, and the basic EDCA access method is adopted as shown in Fig. 5. The backoff counter is $B I$ and the contention window size is $C W$. The station shall select a $B I$ randomly in $[0, C W-1]$ before a new transmission. Thus,

$$
\mathrm{E}[B I]=\frac{C W-1}{2} .
$$




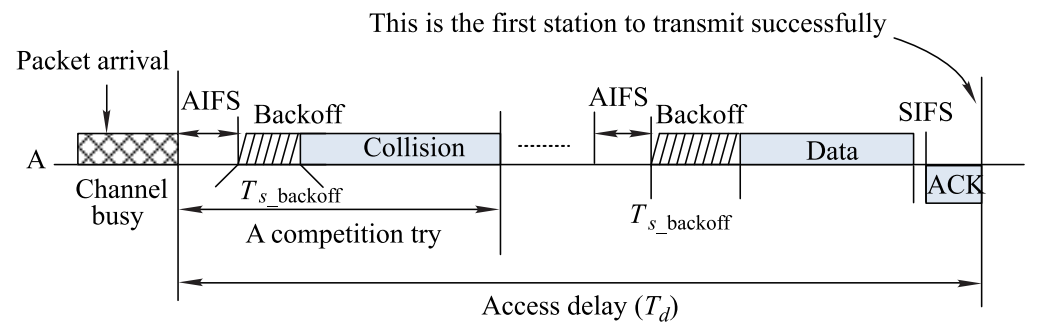

Fig. 5 A success medium access process of one 802.11e station

In this section, $T_{s_{-} \text {backoff }}$ denotes the number of consecutive idle backoff slots in a competition try; in other words, it is the minimal backoff counter of all the stations. $P_{s}$ denotes the probability that one station transmits successfully in a competition try, and $P_{s}(k)$ denotes the probability with an additional condition that $T_{s_{-} \text {backoff }}=k$. For the convenience of presentation, unless otherwise specified, all the time parameters in this section are expressed as multiples of slot, which is a predefined value in the PHY layer. E[.] indicates the expectation of the variable, and $P\{\cdot\}$ indicates the probability of the condition. Formulized definitions are shown as follows:

$$
\left\{\begin{array}{l}
P_{s}(k)=\frac{M}{B I+1}\left(\frac{B I-k}{B I+1}\right)^{M-1} \\
\mathrm{E}\left[T_{s_{-} \text {backoff }}\right]=\sum_{k=0}^{B I} k \cdot P_{s}(k)= \\
\frac{M}{(B I+1)^{M}} \sum_{k=0}^{B I}(B I-k) k^{M-1} \\
\mathrm{E}\left[P_{s}\right]=\sum_{k=0}^{B I} P_{s}(k)=\frac{M}{(B I+1)^{M}} \sum_{k=0}^{B I} k^{M-1}
\end{array}\right.
$$

From the sum formula of the idempotent series, we get

$$
\sum_{k=0}^{B I} k^{M-1} \approx \frac{(B I+1)^{M}}{M}-\frac{(B I+1)^{M-1}}{2} .
$$

Thus, $\mathrm{E}\left[T_{s_{-} \text {backoff }}\right]$ and $\mathrm{E}\left[P_{s}\right]$ can be simplified as

$$
\left\{\begin{array}{l}
\mathrm{E}\left[T_{s_{-} \text {backoff }}\right] \approx \frac{C W+1}{2(M+1)} \\
\mathrm{E}\left[P_{s}\right] \approx \mathrm{E}\left[1-\frac{M}{2(B I+1)}\right]=1-\frac{M}{C W+1} .
\end{array}\right.
$$

Let $T_{d}$ denote the access delay of the first successful transmission among all the stations, including the time spent on the backoff, collisions and packet transmission; $T_{i}$ indicates the value of $T_{d}$ under the condition that the first successful transmission occurs in the $i$ th competition try, and $P_{i}$ indicates the probability of this condition; $S$ is the normalized throughput that is defined as the ratio of the
MAC payload transmission time to the whole access delay. AIFS and SIFS are the parameters defined in the 802.11e Standard and listed in Table 2. According to the definitions, we get

$$
\left\{\begin{array}{l}
P_{i}=\left[1-P_{s}\right]^{i-1} \cdot P_{s} \\
T_{i}=i \cdot\left(\text { AIFS }+\mathrm{E}\left[T_{s_{-} \text {backoff }}\right]+T_{\text {data }}+\text { SIFS }+T_{\text {ack }}\right) \\
T_{d}=\sum_{i=1}^{\infty} P_{i} \cdot T_{i} \\
S=T_{\mathrm{MPDU}} / T_{d}
\end{array}\right.
$$

where $T_{\text {data }}$ is the aggregate transmission time of the PHY overhead $T_{\text {phy_header }}$ and the MAC payload $T_{\mathrm{MPDU}}$.

Define

$$
\left\{\begin{array}{l}
q=\mathrm{AIFS}+T_{\mathrm{data}}+\mathrm{SIFS}+T_{\mathrm{ack}} \\
\varphi=T_{\mathrm{MPDU}} / q
\end{array},\right.
$$

and substitute it into (7), we get

$$
\mathrm{E}\left[T_{d}\right]=\mathrm{E}\left[\sum_{i=1}^{\infty} P_{i} \cdot T_{i}\right]=\frac{(C W+1) / 2(M+1)+q}{1-M /(C W+1)} .
$$

To get the minimal value of $\mathrm{E}\left[T_{d}\right]$, set $\frac{\partial \mathrm{E}\left[T_{d}\right]}{\partial C W}=0$ and solve the equation, we get the optimal CW size:

$$
C W_{\mathrm{opt}} \approx\lceil 1+\sqrt{2 q+1}\rceil \cdot M .
$$

Substitute (9) into (8) and (7), we get the theoretic minimal access delay and maximal throughput as the following:

$$
\left\{\begin{array}{l}
\mathrm{E}\left[T_{d}\right]_{\min } \approx(1+\sqrt{2 q+1})^{2} / 2 \\
S_{\max } \approx \frac{2 \varphi q}{(1+\sqrt{2 q+1})^{2}}
\end{array} .\right.
$$

When the CW size is set properly, the maximal throughput can be achieved. However, according to (9), the optimal CW size increases linearly to the active stations number, which varies dramatically and is difficult to detect in the real networks.

Fortunately, when we substitute (9) into (6), a static parameter of the optimal state is obtained:

$$
T_{s_{-} \text {backoff_opt }} \approx\lceil 1+\sqrt{2 q+1}\rceil / 2 .
$$


In other words, the optimal number of consecutive idle backoff slots does not depend on the active stations number. This is the basis of the proposed algorithm, which is named the multi-priority $\mathrm{CW}$ tuning (MPCT) and presented in Fig. 6.

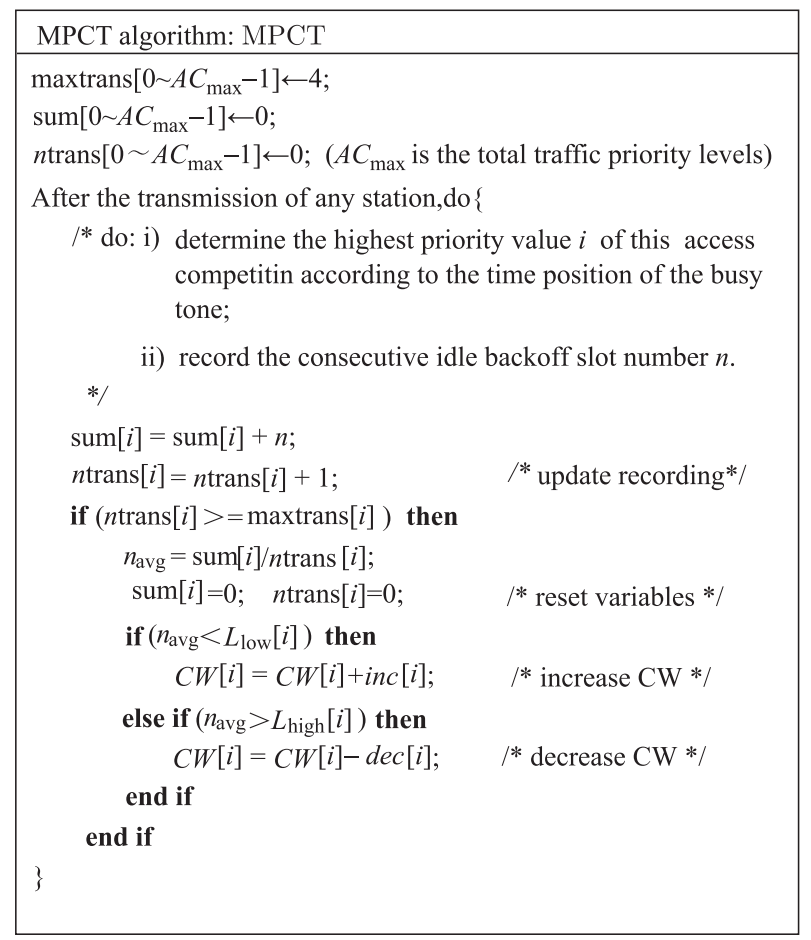

Fig. 6 CW tuning procedure of MPCT algorithm

As shown in Fig. 6, the stations shall always observe the channel for determining the highest priority value $i$ and recording the consecutive idle backoff slots number $n$ in this competition try. The highest priority value can be achieved according to the time position of the BT as shown in Fig. 1 and (1). If a BT is detected at the $k$ th slot, where $k=\operatorname{AIFSN}[i]$, after the channel becomes idle, the highest priority value will be $i$.

maxtrans is a smoothing factor that is used to avoid intensive oscillation of CW sizes, and $n_{\text {avg }}$ is the average number of the consecutive idle backoff slots in maxtrans competition tries. Referring to (11), the expected number of consecutive idle backoff slots is $\lceil 1+\sqrt{2 q+1}\rceil / 2$. Hence, it is easy to think of a way to adjust CW by using the expected value as a trigger threshold. However, to reduce the oscillation of $\mathrm{CW}$ tuning, this paper prefers a double thresholds method, with the parameters setting shown in (12). If $n_{\text {avg }}$ is smaller than $L_{\text {low }}$, increase CW, and if larger than $L_{\text {high }}$, decrease it. To be mentioned that, all these parameters are arrays with individual values for each AC.

$$
\left\{\begin{array}{l}
L_{\text {low }}=\lceil 1+\sqrt{2 q+1}\rceil / 2-1 \\
L_{\text {high }}=\lceil 1+\sqrt{2 q+1}\rceil / 2+1
\end{array}\right.
$$

The adjustment steps inc and dec are another trade-off factors of the convergence speed and stability for the proposed algorithm. Larger adjustment step increases the convergence speed, but suffers from worse stability. Therefore, we introduce a step coefficient to dynamically adjust the increment and decrease step. When the deviation from the optimal value is far away, the adjustment step is increased to speed up convergence; when the deviation from the optimal value is near, the adjustment step is reduced to improve stability.

$$
\left\{\begin{array}{l}
\text { inc }=\lceil 1+\sqrt{2 q+1}\rceil\left\lceil L_{\mathrm{low}} / n_{\mathrm{avg}}\right\rceil \\
\text { dec }=\lceil 1+\sqrt{2 q+1}\rceil\left\lceil n_{\mathrm{avg}} / L_{\mathrm{high}}\right\rceil
\end{array}\right.
$$

\section{Simulation result}

In this section, the performance of the proposed scheme is evaluated through Opnet simulations. The EDCA [3], DPCA [23] and PIS [14] are also implemented for comparison. Table 2 shows the common parameters picked up from the 802.11e Standard, and Table 3 shows the individual parameters similar to [29] and [30].

Table 2 Common parameters

\begin{tabular}{cc}
\hline Parameter & Value \\
\hline Bandwidth/modulation & $20 \mathrm{MHz} / \mathrm{OFDM}$ \\
Bit-rate/Mbps & 54 \\
Slot/ $\mu \mathrm{s}$ & 9 \\
SIFS/ $\mu \mathrm{s}$ & 16 \\
AIFS & SIFS+AIFSN $\cdot$ slot \\
PHY overhead/ $\mu$ s & 20 (Preamble+PHY header) \\
MAC overhead/Octets & 40 (MAC header+FCS ) \\
Data payload size/Octets & $\leqslant 2048$ \\
ACK payload size/Octets & 14 \\
Retransmit limit & 7 \\
\hline
\end{tabular}

Table 3 Individual parameters

\begin{tabular}{ccccc}
\hline \multicolumn{2}{c}{ Parameter } & AC_VO & AC_VI & AC_BE \\
\hline \multirow{3}{*}{ EDCA } & AIFSN & 2 & 3 & 6 \\
& $C W_{\min }$ & 4 & 8 & 16 \\
& $C W_{\max }$ & 8 & 16 & 1024 \\
\hline \multirow{6}{*}{ PIS } & AIFSN & 2 & 2 & 2 \\
& $C W_{\min }$ & 32 & 32 & 32 \\
& $C W_{\max }$ & 256 & 1024 & 1024 \\
& $n^{a}$ & 2.3 & 3.1 & 3.9 \\
\hline \multirow{4}{*}{ DPCA } & AIFSN & 2 & 3 & 4 \\
& $C W_{\min }$ & 32 & 32 & 32 \\
& $C W_{\max }$ & 256 & 1024 & 1024 \\
\hline \multirow{6}{*}{ PABT } & AIFSN & 2 & 3 & 4 \\
& $C W_{\min }$ & 32 & 32 & 32 \\
& $C W_{\max }$ & 256 & 1024 & 1024 \\
& $L_{\text {low }}$ & 3 & 5 & 6 \\
& $L_{\text {high }}$ & 4 & 6 & 7 \\
& $a, b$ & 6 & 10 & 12 \\
\hline
\end{tabular}

In these simulations, stations are randomly distributed in an area of $150 \mathrm{~m}$ by $150 \mathrm{~m}$. Each station has three traffic 
flows, i.e., AC_VO, AC_VI and AC_BE, one for each type with the parameters shown in Table 4. The G.729a is supposed to be the voice encoder. It encodes $10 \mathrm{~ms}$ voice data into a block of 10 octets, and combines four blocks into one packet. By adding 40 octets IP/UDP/RTP headers, one 80 octets voice over IP (VOIP) frame is constructed. The H.264 is supposed to be the video encoder for the common intermediate format (CIF) with the detailed parameters shown in Table 4. Each station has a saturated AC_BE traffic flow that always has a packet to send.

Table 4 Traffic related parameters

\begin{tabular}{cccc}
\hline Parameter & AC_VO & AC_VI & AC_BE \\
\hline Encoder & G.729a & H.264 & none \\
Frame length/octets & 80 & 1350 & 1500 \\
Inter-arrival time/ms & 40 & 10 & 1 \\
Buffer size/packets & 64 & 64 & 64 \\
MAC data rate/Mbps & 0.024 & 1.112 & 12.32 \\
QoS for delay/ms & $<100$ & $<200$ & none \\
QoS for PLR/\% & $<1$ & $<1$ & none \\
\hline
\end{tabular}

The performance metrics of interest include traffic capacity, throughput, fairness, delay and PLR. Traffic capacity is the maximal number of flows whose QoS requirements can be concurrently fulfilled. Throughput is the transmission bit-rate including the IP and MAC overhead. Delay is the period from the generating of the packet to its successful transmission, which includes the time spent on local queuing, retransmission and acknowledgment. Fairness is evaluated by the fairness index $F I$ as follows:

$$
F I=\frac{\left(\sum_{i=1}^{M} S_{i}\right)^{2}}{M \cdot \sum_{i=1}^{M}\left(S_{i}\right)^{2}}
$$

where $S_{i}$ is throughput of the station $i$, and $M$ is the number of stations. Higher FI implies better fairness, and the upper-bound of $F I$ is 1 . PLR is the ratio of the dropped packet number to the generated packet number. In the simulations, packets are dropped due to three reasons: (i) unacceptable transmission delay; (ii) collisions and bit errors; (iii) packet buffer overflows.

The International Telecommunication Union (ITU) G.114 recommends maximal 150 ms one-way delay and $1 \%$ PLR for voice calls [33]. Considering both transmission delay in the wired network and coding time, we set the maximal allowable delay to $100 \mathrm{~ms}$ in these simulations. Any voice call that does not fulfill these QoS requirements will be dropped. A similar setting for the video traffic is adopted except that the maximal allowable delay is set to $200 \mathrm{~ms}$ [9]. Thus, we can achieve the voice and video traffic capacities supported by the network.
First, we estimate the theoretical traffic capacity through the model presented in Section 3. We get that the expected access delay of AC_VI is about $391 \mu$ s according to (10), and the packet arrival rate of $\mathrm{AC}_{-} \mathrm{VI}$ is $10 \mathrm{~ms}$ according to Table 3. Diving $10 \mathrm{~ms}$ by $391 \mu$ s, we assert that the traffic capacity for AC_VI will be no more than 25 . Similar calculations can be done to get the capacity of AC_VO, and the result is about 228 .

Fig. 7 gives the simulation results of the traffic capacities for different schemes. Due to its intrinsic problems described in Section 1, the EDCA can not even fulfill the QoS requirements of five voice flows. The DPCA performs a little better, while the PIS produces another remarkable improvement. The proposed PABT can fulfill the QoS of all the AC_VO flows even when the stations number reaches 100 , which is about 2 times higher than the PIS algorithm and 20 times higher than the 802.11e EDCA algorithm. Moreover, the simulation result of the AC_VI capacity is 22. This value is a little less than the theoretical value 25 , because the theoretical value is calculated for the single $\mathrm{AC}$ scenario, but the simulations also introduce the $\mathrm{AC}$-VO and AC_BE traffic flows, which will also occupy part of the bandwidth.
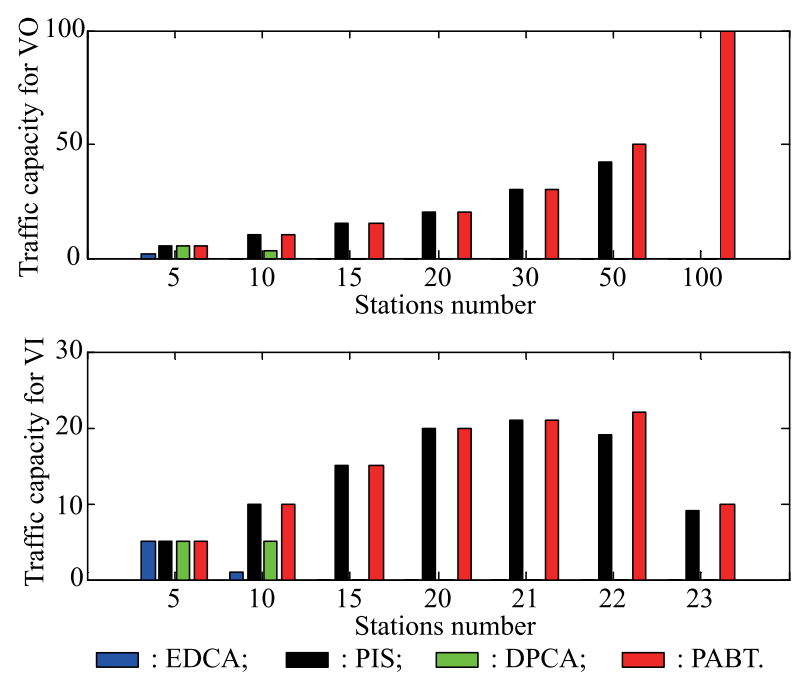

Fig. 7 Comparison of the real-time traffic capacity

Secondly, the individual and aggregate throughput of the traffic is compared in Fig. 8. The aggregate throughput of EDCA degrades sharply due to the severe collisions as the stations number increases, and the absolute priority access cannot be guaranteed. As mentioned above, the traffic capacities of AC_VO and AC_VI are both less than or equal to 5. However, as shown in Fig. 8, the AC_BE traffic still occupies part of the bandwidth even when the QoS requirements of AC_VO and AC_VI traffic have not been fulfilled. The DPCA performs better, because it uses the 
BT method to differentiate the traffic, and thus can optimize the $\mathrm{CW}$ settings individually. The aggregate throughput performance of PIS is much better, since it also adopts an optimal CW tuning algorithm. The PABT performs the best, due to not only the higher throughput, but also the absolute priority grantee that the LP traffic can scarcely access channels before the QoS requirements of the HP traffic have been fulfilled.

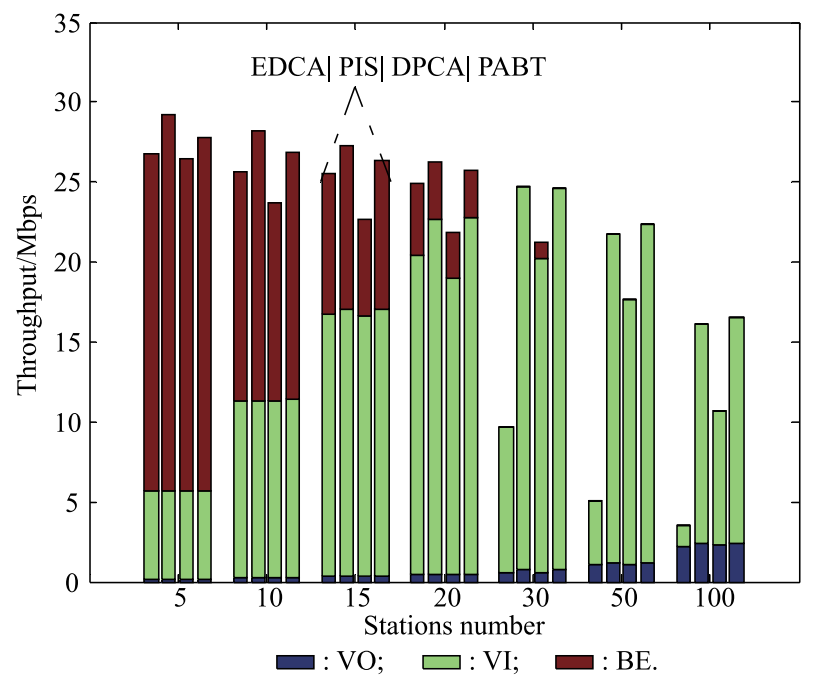

Fig. 8 Comparison of the throughput

Thirdly, the fairness performance is presented in Fig. 9. In this simulation, $F I$ is computed every $2 \mathrm{~s}$. The fairness indices of the PIS and PABT are both much better than the EDCA and DPCA, especially when the stations number increases. Last but not least, the superiorities of the proposed PABT over others are also shown in Fig. 10 and Fig. 11 through the delay and PLR performance. To be mentioned that, since the traffic capacity of AC_VI is only 22 , so, if the stations number exceeds this value, the performance of PABT will decline sharply.

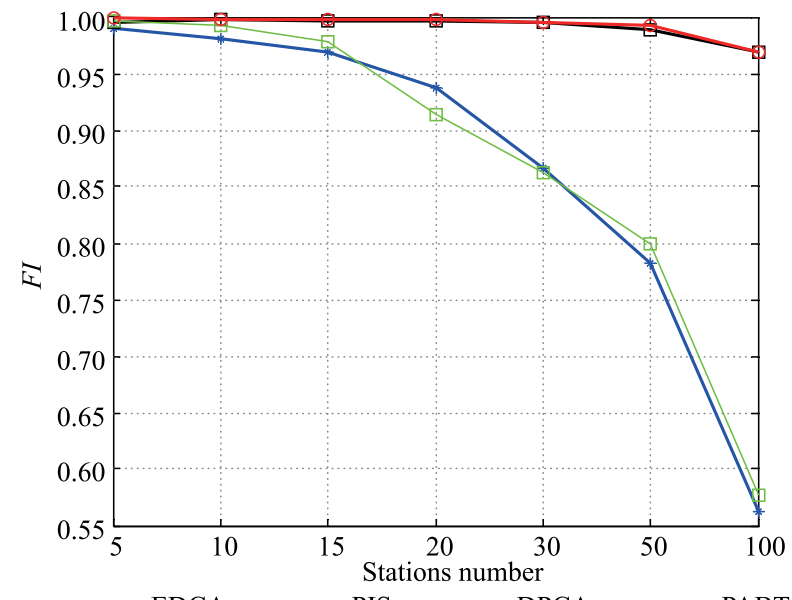

$\longrightarrow:$ EDCA; $\square:$ PIS; $\square:$ DPCA; $\multimap:$ PABT

Fig. 9 Comparison of the fairness index for AC_BE
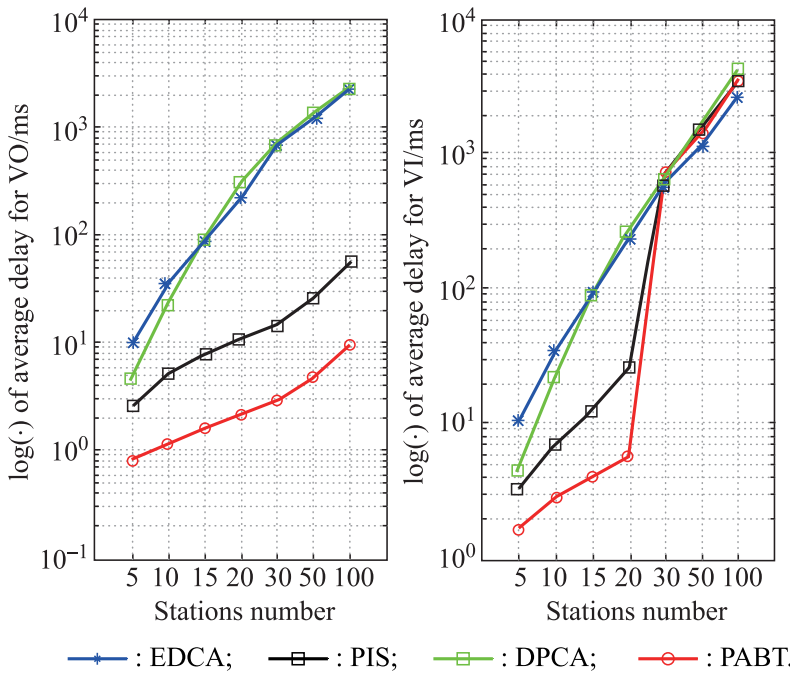

Fig. 10 Comparison of average delay for real-time traffic
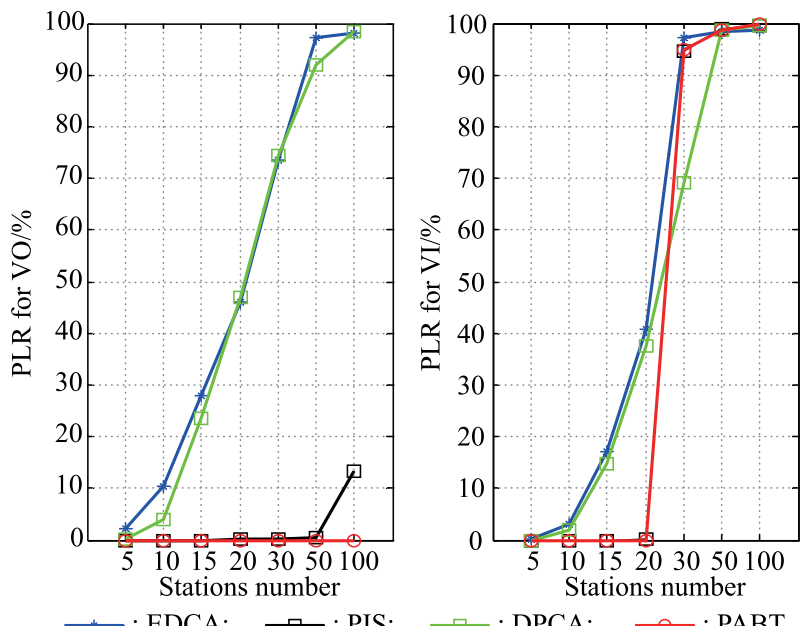

Fig. 11 Comparison of PLR for real-time traffic

Another important feature of the proposed scheme is the fast convergence speed after a sudden change of the active stations number. In this scenario, five greedy stations competing for the channel at the beginning; $10 \mathrm{~s}$ later, five more stations start competing for the channel, and leave after $5 \mathrm{~s}$. Fig. 12 shows the $\mathrm{CW}$ trace of a random selected besteffort traffic flow in the network. It shows that PABT will converge to a new stable state in $0.2 \mathrm{~s}$.

In summary, the performance of the EDCA and DPCA are not so satisfying, and the PIS performs much better. However, when the stations number is more than 50 , the superiorities of the proposed PABT become more and more clearly.

\section{Conclusions}

In this paper, we propose an access method based on the 802.11e EDCA to improve the QoS performance. The proposed PABT scheme combines the BT reservation and the 
optimal CW tuning schemes together, and solves the uncertain priority access and the limited real-time traffic capacity problems simultaneously. The simulation results show that the proposed scheme can support much more traffic flows with intense QoS requirements, and outperforms the EDCA significantly on throughput, delay and PLR, regardless of the contention levels. Moreover, this scheme can provide both good fairness for the traffic flows with the same priority and guaranteed priority access for the flows with different priorities.

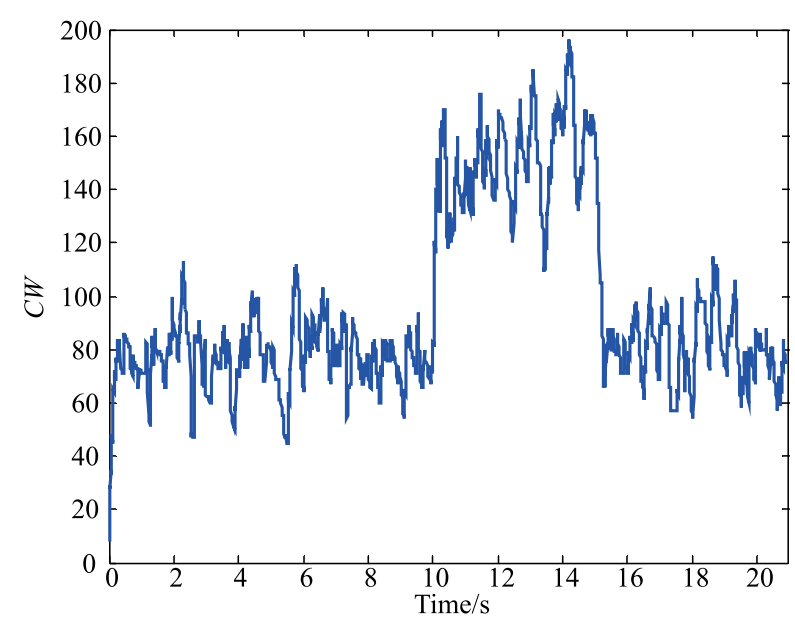

Fig. 12 CW trace after a sudden change of the active stations number

\section{References}

[1] MEEKER M. 2015 Internet trends. KPCB report, https://www.kleinerperkins.com/InternetTrends.

[2] SZOTT K, NATKANIEC M, SZOTT S. What's new for QoS in IEEE 802.11. IEEE Network, 2013, 27(6): 95 - 104.

[3] IEEE Standard 802.11e. Wireless LAN medium access control (MAC) and physical layer specifications, amendment 8: MAC quality of service enhancements, 2005.

[4] PATTARA-ATIKOM W, KRISHNAMURTHY P, BANERJEE S. Distributed mechanisms for quality of service in wireless LANs. IEEE Wireless Communications, 2003, 10(3): 26-34.

[5] CHOU Z T, HUANG C Q, CHANG J M. QoS provisioning for wireless LANs with multi-beam access point. IEEE Trans. on Mobile Computing, 2014, 13(9): 2113-2127.

[6] HU C, HOU J C. A novel approach to contention control in IEEE 802.11e-operated WLANs. Proc. of the 26th IEEE International Conference on Computer Communications, 2007: 1190 - 1198.

[7] PATTARA-ATIKOM W, BANERJEE S, KRISHNAMURTHY P. A-DRAFT: an adaptive QoS mechanism to support absolute and relative throughput in 802.11 wireless LANs. Proc. of the International Conference on Modeling, Analysis and Simulation of Wireless and Mobile Systems, 2004: 117-126.

[8] CHARFI E, CHAARI L, KAMOUN L. PHY MAC enhancements and QoS mechanisms for very high throughput WLANs: a survey. IEEE Communications Surveys \& Tutorials, 2013, 15(4): $1714-1735$.
[9] VAIDYA N H, DUGAR A, GUPTA S, et al. Distributed fair scheduling in a wireless LAN. IEEE Trans. on Mobile Computing, 2005, 4(6): 616-629.

[10] IEEE Standard 802.11aa. Wireless LAN medium access control (MAC) and physical layer specifications, amendment 2: MAC enhancements for robust audio video streaming, 2012.

[11] HAMMOURI M M, DAIGLE J N. A distributed scheduling mechanism to improve quality of service in IEEE 802.11 ad hoc networks. Proc. of the IEEE Symposium on Computers and Communications, 2011: 1-6.

[12] GUPTA A, LIN X, SRIKANT R. Low-complexity distributed scheduling algorithms for wireless networks. IEEE/ACM Trans. on Networking, 2009, 17(6): 1846-1859.

[13] HOFFMANN O, SCHAEFER F M, KAYS R, et al. Prioritized medium access in ad-hoc networks with a SystemClick model of the IEEE 802.11n MAC. Proc. of the 21st International Symposium on Personal, Indoor and Mobile Radio Communications, 2010: 2805 - 2810 .

[14] NASSIRI M, HEUSSE M, DUDA A. A novel access method for supporting absolute and proportional priorities in 802.11 WLANs. Proc. of the 27th IEEE International Conference on Computer Communications, 2008: $1382-1390$.

[15] PARK E, KIM D, CHOI C, et al. Improving quality of service and assuring fairness in WLAN access networks. IEEE Trans. on Mobile Computing, 2007, 6(4): $337-350$.

[16] XIAO Y, LI H, CHOI S. Two-level protection and guarantee for multimedia traffic in IEEE 802.11e distributed WLANs. ACM Wireless Networks, 2009, 15(2): $141-161$.

[17] SAIF A, OTHMAN M, SUBRAMANIAM S, et al. Impact of aggregation headers on aggregating small MSDUs in 802.11n WLANs. Proc. of the International Conference on Computer Applications and Industrial Electronics, 2010: 630-635.

[18] KLEINROCK L, TOBAGI F. Packet switching in radio channels: Part I - carrier sensing multiple-access modes and their throughput - delay characteristics. IEEE Trans. on Communications, 1977, 25(10): 1103-1119.

[19] HAAS Z J, DENG J. Dual busy tone multiple access (DBTMA) - a multiple access control scheme for ad hoc networks. IEEE Trans. on Communications, 2002, 50(6): $975-$ 985.

[20] YANG X, VAIDYA N H. Priority scheduling in wireless ad hoc networks. Proc. of the IEEE International Conference on Mobile Adhoc and Sensor Systems, 2002: 71 - 79.

[21] BANERJEE A, TANTRA J W, FOH C H, et al. A service/device differentiation scheme for contention-tone-based wireless LAN protocol. IEEE Trans. on Vehicular Technology, 2010, 59(8): 3872-3885.

[22] JIANG H, WANG P, ZHUANG W. A distributed channel access scheme with guaranteed priority and enhanced fairness. IEEE Trans. on Wireless Communications, 2007, 6(6): 2114 2125 .

[23] KIM S, HUANG R, FANG Y. Deterministic priority channel access scheme for QoS support in IEEE 802.11e wireless LANs. IEEE Trans. on Vehicular Technology, 2009, 58(2): $855-864$.

[24] ZHOU X, ZHENG C. In-band busy tone protocol for QoS support in distributed wireless networks. Proc. of the 16th International Conference on Computational Science and Its Applications, 2016: $555-562$. 
[25] BIANCHI G. Performance analysis of the IEEE 802.11 distributed coordination function. IEEE Journal on Selected Areas in Communications, 2000, 18(3): 535-547.

[26] CALI F, CONTI M, GREGORI E. Dynamic tuning of the IEEE 802.11 protocol to achieve a theoretical throughput limit. IEEE/ACM Trans. on Networking, 2000, 8(1): 785-799.

[27] HEUSSE M, ROUSSEAU F, GUILLIER R, et al. Idle sense: an optimal access method for high throughput and fairness in rate diverse wireless LANs. Proc. of the International Conference on the Applications, Technologies, Architectures, and Protocols for Computer Communication, 2005: 121 - 132.

[28] YU Q, ZHUANG Y, MA L. Dynamic contention window adjustment scheme for improving throughput and fairness in IEEE 802.11 wireless LANs. Proc. of the IEEE Global Communications Conference, 2012: 5074-5080.

[29] ZHOU X, ZHENG C W. Busy tone based prioritized access for distributed wireless networks. Proc. of the 17th IEEE International Symposium on a World of Wireless, Mobile and Multimedia Networks, 2016: 1-3.

[30] ZHOU X, ZHENG C, LIAO M. Full-feedback backoff algorithm for distributed wireless networks. Proc. of the International Wireless Communications and Mobile Computing Conference, 2015: $1079-1084$.

[31] GAO Y, SUN X, DAI L. IEEE 802.11e EDCA networks modeling, differentiation and optimization. IEEE Trans. on Wireless Communications, 2017, 13(7): 3863 - 3879.

[32] QIAO D, SHIN K. Achieving efficient channel utilization and weighted fairness for data communications in IEEE 802.11 WLAN under the DCF. Proc. of the IEEE International Workshop on QoS, 2002: 227-236.

[33] ITU-T Standard G.114. One-way transmission time, 2003.

\section{Biographies}

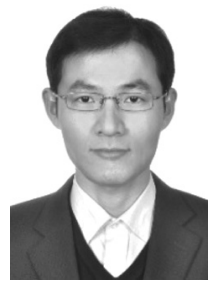

ZHOU Xin was born in 1986. He received his B.S. degree in electronic engineering from Huazhong University of Science and Technology, China, in 2007, M.S. degree in communication and information system from Huazhong University of Science and Technology in 2009, and Ph.D. degree in computer sciences from the University of Chinese Academy of Sciences in 2017. He has worked in the Institute of Software, Chinese Academy of Sciences since 2009, and has been an associate research fellow since 2017. He participated in the design and development of the first VHF/UHF military cognitive radio of China, and won one second class prize of Military Science and Technology Progress Award. His current research interests include ad hoc networks, cognitive radio networks and sepctrum recognition.

E-mail: zhouxin@iscas.ac.cn

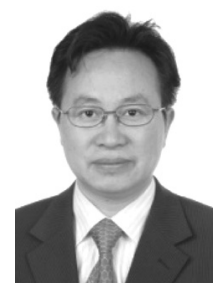

ZHENG Changwen was born in 1969. He received his B.S. degree in mathmatics from Central China Normal University, Wuhan, China, in 1992, M.S. degree in economics from Huazhong University of Science and Technology in 1998, and Ph.D. degree in image processing and artificial intelligence from Huazhong University of Science and Technology in 2003. He has been a professor in Institute of Software, Chinese Academy of Sciences since 2005, and the vice director of the National Key Laboratory of Integrated Information System Technology since 2010. He is the senior member of China Computer Federation and Chinese Society of Astronautics. His research interests include computer networks, route planning for air vehicles, system simulation, and artificial intelligence.

E-mail: changwen@iscas.ac.cn 